\title{
Influência do local de análise dopplervelocimétrica na artéria cerebral média
}

\author{
The influence of the site of middle cerebral Doppler flow analysis
}

\author{
Marco Aurélio Martins de Souza ${ }^{1}$, Hubert Caldeira ${ }^{2}$, Rosamaria Ribeiro Caracas ${ }^{3}$, Leonardo Biondi ${ }^{3}$, \\ Richard Duarte ${ }^{3}$, Flávia Pollyana Almeida Prates ${ }^{3}$
}

\section{RESUMO}

Objetivo: avaliar se existem diferenças entre índices os ovários dopplervelocimétricos em função do local de insonação da artéria cerebral média em pacientes saudáveis comparando-as a outro grupo com intercorrências. Métodos: estudo prospectivo, randomizado, desenvolvido no período de junho de 2003 a março de 2004. Foram analisados os índices doplervelocimétricos de 100 pacientes: grupo de estudo (GE, $\mathrm{n}=50$ ): pacientes internadas tendo como critérios de inclusão: estar entre a $28^{\mathrm{a}}$ e a $34^{\mathrm{a}}$ semana de gestação e diagnóstico de hipertensão arterial crônica materna, pré-eclampsia ou restrição de crescimento intrauterino (CIUR). Como grupo controle (GC), 50 pacientes saudáveis, grávidas entre a $28^{\mathrm{a}}$ e a $34^{\mathrm{a}}$ semana. As variáveis doplervelocimétricas analisadas foram o índice de resistência (IR), o índice de pulsatilidade (IP) e a relação sístole/diástole (SD). Todos os três índices foram obtidos e em dois locais diferentes: a primeira medida em região do diencéfalo, logo após o início da artéria cerebral média, e a segunda em localização mais distal, no telencéfalo. Resultados: As medianas dos índices no GE na primeira e segunda medida foram respectivamente para o IP de 1,55 e 1,69; para o IR de 0,77 e 0,79; para o SD de 4,29 e 4,86. No GC os valores foram para o IP de 1,73 e 1,86; para o IR de 0,83 e 0,79 e para o SD de 5,83 e 5,46. Não houve diferenças entre os locais, com valor p de 0,38; 0,29 e 0,39, respectivamente para o IP, IR e SD. Em 15 fetos centralizados, a mediana dos índices no diencéfalo foi IP=1,02; IR=0,63 e SD=2,68. No telencéfalo a mediana foi IP=0,95; IR=062 e $\mathrm{SD}=2,44$. Não houve diferenças entre os dois locais, com valor p de 0,53 para o IP; 0,56 para o IR e 0,31 para o SD. Conclusão: O local de insonação não interfere nos valores dos índices de doplervelocimetria nas artérias cerebrais médias.

PALAVRAS CHAVES: Fluxometria por laser-doppler; Artérias cerebrais; Hipertensão; Desenvolvimento embrionário e fetal

\section{ABSTRACT}

Purpose: to evaluate if there is any difference between Doppler indexes in the middle cerebral artery in two different sites of insonation in healthy patients and in patients with diseases. Methods: a random prospective survey, in the period from June 2003 to March 2004 that analyzed the Doppler indexes of 100 patients: patient group $(n=50)$ included patients admitted to Clemente Farias University Hospital, which is part of UNIMONTES-MG, havinfg as inclusion criteria: to be in the 28th to 34th gestational week, diagnosis of chronic arterial hypertension, pre-eclampsia, intrauterine growth restriction. As control group, 50 healthy pregnant patients between the 28th and the 34th week, originary from SEMESP's clinic. The Doppler variables were the resistance index (RI), the pulsatility index (PI) and the relation systole/diastole (SD). All three Doppler indexes were assessed at two different sites of the cerebral artery: the first measurement in the diencephalons region, soon after the beginning of the middle cerebral artery and the second on a distal location in the telencephalon. The median Doppler indexes in the patient group in the first and second measurements were 1.55 and 1.69 for the PI, 0.77 and 0.79 for RI and 4.29 and 4.86 for SD, respectively. In the control group, the values were 1.73 and 1.86 for the PI; 0.83 and 0.79 for RI and 5.83 and 5.46 for SD. There were no differences between sites with a p value of $0.38,0.29$ and 0.39 for PI, RI and SD, respectively. In $15^{\mathrm{t}}$ fetuses with centralization (brain sparing effects), in the diencephalon the median indexes were 1.02 for PI, 0.63 for RI and 2.68 for SD. In the epencephalon the median indexes were 0.95 for IP, 0.62 for RI and 2.44 for SD. There were no differences between sites, with a $\mathrm{p}$ value of 0.53 for PI; 0.56 for IR and 0.31 for SD. The Doppler index site of assessment in the middle cerebral arteries does not interfere with the results.

KEYWORDS: Laser doppler-flowmetry; Middle cerebral arteries; Hypertension; Embryo and fetal development

\footnotetext{
Hospital Universitário Clemente Faria, Maternidade Maria Barbosa, Universidade Estadual de Montes Claros, UNIMONTES (MG).

1 Professor Adjunto.

2 Coordenador da Maternidade Maria Barbosa.

3 Médicos Residentes de Ginecologia e Obstetrícia.

Correspondência: Marco Aurélio Martins de Souza

Av. Dep. Esteves Rodrigues 616 - sl 203 - Centro - 39400-215 - Montes Claros - MG - Telefone: 0xx(38) 3222-1771 - e-mail: marcoams@uai.com.br Recebido em: 21/5/2004

Aceito com modificações em: 30/3/2005
} 


\section{Introdução}

A investigação dopplervelocimétrica da circulação fetal representa marco importante para a monitorização de fetos com crescimento intrauterino restritos, anemia, hidropisias gemelares, gravidezes com insuficiência placentária, bem como em várias outras circunstâncias, e desse modo pode ajudar a determinar o momento ideal para o parto ${ }^{1}$.

Na literatura, existem poucos estudos que avaliam se o local de análise das artérias cerebrais médias, influenciam na obtenção dos indices Doppler. Por ser artéria de grande importância no estudo da hemodinâmica fetal, é fundamental analisar se modificações técnicas podem alterar de maneira significativa os resultados auferidos.

A análise da artéria cerebral média é importante na prática obstétrica, eventualmente definindo o momento da interrupção de uma gravidez (centralização). Estudos que possam avaliar se existem modificações nos índices dopplervelocimétricos em função das técnicas de exame são sempre promissores para afiançar a segurança, confiabilidade e reprodutibilidade do método ${ }^{2}$. As diferentes regiões irrigadas pela artéria cerebral média podem ter comportamentos diversos em relação à hipóxia tecidual, não sendo o fenômeno da centralização cronologicamente universal. Há evidências de que o córtex é o primeiro local a ser poupado, para depois se pouparem os centros mais inferiores. Assim, a vasodilatação ocorreria em segmentos distintos deste vaso ${ }^{3}$.

O objetivo do presente estudo prospectivo foi averiguar se existem modificações nos îndices de dopplervelocimetria da artéria cerebral média, em função do local de obtenção das variáveis de interesse: índice de resistência (IR), índice de pulsatilidade (IP) e relação sístole/diástole (SD).

\section{Pacientes e Métodos}

Após aprovação pelo Comitê de Ética e Pesquisa da Universidade Estadual de Montes Claros, no período de junho de 2003 a março de 2004 analisaram-se os dados dopplervelocimétricos de 50 pacientes grávidas, com idade gestacional entre a $28^{\mathrm{a}}$ e a $34^{\mathrm{a}}$ semana de gestação, formando o grupo estudo. Como critérios de inclusão, deveriam assinar o termo de consentimento pós-informado sobre a pesquisa, estar na idade gestacional citada, e a indicação do exame ser a doença hiperten- siva especifica da gestação (DHEG), ou hipertensão arterial sistêmica ou o crescimento intrauterino restrito (CIUR). Todas deveriam ser atendidas no Hospital Universitário Clemente Farias, Maternidade Maria Barbosa, Universidade Estadual de Montes Claros (UNIMONTES-MG). Este grupo estudo foi subdividido em exames normais $v s$ anormais (centralização) para comparações em uma mesma população (doença). Como grupo controle, selecionaram-se 50 pacientes saudáveis da clinica SEMESP, com idade gestacional entre a $28^{\mathrm{a}}$ e a $34^{a}$ semana de gestação, vindo de demanda espontânea ao serviço.

A seleção das pacientes no grupo estudo (internadas) foi realizada com distribuição amostral probabilística por meio da geração de séries aleatórias em programa de computador, onde para os 10 leitos disponiveis selecionavam-se as pacientes que preenchiam os critérios de inclusão no estudo e distribuiam-se senhas (a, b, c...). Após esta pré-seleção dos disponíveis, eram gerados os escolhido, pela combinação das duas possibilidades, exame sim, exame não. A série aleatória a realizar o ultra-som era então encaminhada à clínica SEMESP. A seleção no grupo controle obedecia aos critérios de amostragem probabilística mediante escolha aleatória do exame que iria para o grupo controle, obedecendo aos critérios de inclusão. A seqüência gerada em computador definia, dentre os disponíveis, qual o laudo que iria para o grupo controle. O exame tanto no grupo controle como no de alto risco foi realizado pelo mesmo profissional, no mesmo período do dia, sempre pela manhã, tentando assim, evitar efeitos do ciclo circardiano e interobservador ${ }^{4}$.

Todos os exames foram realizados no mesmo equipamento da marca Medison Sonoacer 9900 3D (Coréia, 2003) com a sonda convexa de freqüência variável 3,5 a $5 \mathrm{MHz}$. Os índices dopplervelocimétricos da artéria cerebral média foram obtidos com o uso de varredura axial da cabeça fetal ao nível do tálamo e cavo do septo pelúcido. Em seguida movia-se o transdutor até observar o poligono de Willis e a pulsação das duas artérias cerebrais médias. A análise espectral era feita na artéria anteriormente localizada. Calibrava-se o indicador de amostra para um volume de $1,0 \mathrm{~mm}^{3}$ e tomavam-se as medidas nos dois locais propostos: primeiramente logo após a sua origem na carótida interna, portanto na região do diencéfalo, e o segundo exame em sua região mais distal, localizada no córtex, em área do telencéfalo, próximo da calota craniana.

A correção do ângulo entre o feixe sonoro e o fluxo sangüineo era feita entre $0^{\circ}$ e $10^{\circ}$. O filtro era ajustado na freqüência de $50-100 \mathrm{~Hz}$, para evi- 
tar a perda do componente diastólico. Em cada um dos exames dopplervelocimétricos, eram definidas as variáveis de interesse: IR, IP e SD. Os valores de cada eram obtidos pela análise dos melhores traçados gráficos das ondas dos respectivos vasos, escolhidos pelo operador, e o aparelho os processava automaticamente com seu software. O valor final era obtido pela média aritmética de três medidas ideais.

Tanto no grupo estudo (DHEG, hipertensas, CIUR) quanto no grupo controle saudáveis, os dados obtidos eram tabulados diretamente no programa de computador com o software Epi-Info $2000^{5}$ e analisados em estatística descritiva, usandose, conforme as variáveis de interesse, o teste $t$ de Student (paramétrico) e os não paramétricos Mann-Whitney ou Mantel-Haenszel para nivel de significância de $95 \%(p<0,05)$. Foram comparados os índices dopplervelocimétricos proximal e distal da artéria cerebral média dentro dos mesmos grupos e entre eles. Ressalte-se que o grupo estudo (doenças) foi subdividido em exames normais $v s$ anormais (centralizados ou com CIUR) comparados entre si e com os fetos sem centralização. Desta forma, foi possivel avaliar se existem modificações em função do local, nos casos em que a auto-regulação está presente (fetos centralizados).

\section{Resultados}

A idade média foi de $25 \pm 3$ anos (grupo estudo) e de $28 \pm 4$ anos (grupo controle). A paridade foi de $2 \pm 2$ filhos (grupo estudo) e de $3 \pm 2$ filhos (grupo controle), ( $p=0,73)$. Todos os três índices foram obtidos nos dois locais estipulados, para os 100 fetos. Os dois grupos mostraram vários critérios de homocedasticidade (igualdade de variâncias), portanto, foram considerados comparáveis, apropriando o teste $t$ de Student e a ANOVA No critério idade gestacional observou-se média de 33 semanas (grupo controle) e de 33 semanas (grupo estudo) ( $p=0,99$; ANOVA, $\chi^{2}=0,21$, d.f. $\left.=1, p=0,63\right)$.

A espessura placentária média foi de $36 \pm 3$ $\mathrm{mm}$ (grupo controle) e de $35 \pm 4 \mathrm{~mm}$ no grupo estudo ( $\mathrm{p}=0,85$, Mantel/Haenszel) com o mínimo de 20 $\mathrm{mm}$ neste. Avaliando o grau da placenta, verificou-se, nos casos de DHEG e/ou CIURs, amadurecimento mais precoce, com classificação grau II ou III de Grannum em 32 pacientes, ao passo que no grupo controle o amadurecimento foi grau II ou III em apenas 15 , mostrando diferenças com nivel de significância estatística ( $p=0,04$; MantelHaenszel, OR: 1,26; IC 95\%: 1,01 a 1,57). Como a classificação placentária é critério subjetivo, com o exame sendo feito pelo mesmo observador, reduziu-se o erro de classificação que poderia ser verificado interobservador.

Não se observou nenhum caso de oligoâmnio no grupo controle e 5 casos no grupo estudo, mostrando diferença estatisticamente significativa $(\mathrm{p}=0,02 ;$ Mann-Whitney). A freqüência cardíaca fetal não mostrou alterações significativas, com média no grupo estudo de $132 \mathrm{bpm}$ e de $140 \mathrm{bpm}$ no grupo controle ( $\mathrm{p}=0,58$; Mann-Whitney). A centralização e/ou CIUR esteve presente em 30\% do grupo estudo $(\mathrm{n}=15)$ e em $0,6 \%(\mathrm{n}=3)$ no grupo controle ( $p=0,02$; OR: 4,33; IC 95\%: 1,06-20,51). A DHEG foi diagnosticada em $80 \%(n=40)$ do grupo estudo e em nenhum caso no grupo controle. A hipertensão arterial crônica correspondeu aos outros 20\% das indicações no Grupo Estudo.

$\mathrm{Na}$ estatística descritiva dos dados dopplervelocimétricos utilizou-se como medida de tendência central a mediana que, por ser robusta, é mais apropriada para as comparações em trabalhos com indices. As medianas dos índices para a cerebral no grupo estudo na primeira e segunda medida foram respectivamente para o IP de 1,55 e 1,69; para o IR de 0,77 e 0,79; para o SD de 4,29 e 4,86. No grupo controle os valores foram para o IP de 1,73 e 1,86; para o IR de 0,83 e 0,79 e para o SD de 5,83 e 5,46 (Tabela 1). As análises estatísticas destas diferenças, quando comparadas entre os dois grupos, não mostraram modificações significativas, conforme Tabela 1, com um valor $\mathrm{p}$ de 0,38, 0,29 e 0,39, respectivamente para o IP, IR e SD.

Tabela 1 - Comparação dos índices dopplervelocimétricos da artéria cerebral em dois 'locais no grupo estudo (DHEG; CIUR) e grupo controle.

\begin{tabular}{lcccccc}
\hline \multicolumn{1}{c}{$\begin{array}{c}\text { Índices } \\
\text { dopplervelocimétricos }\end{array}$} & \multicolumn{2}{c}{$\begin{array}{c}\text { Grupo estudo } \\
\mathrm{n}=\mathbf{5 0}\end{array}$} & \multicolumn{2}{c}{$\begin{array}{c}\text { Grupo controle } \\
\mathrm{n}=\mathbf{5 0}\end{array}$} \\
& $\begin{array}{c}\text { Região } \\
\text { proximal }\end{array}$ & $\begin{array}{c}\text { Região } \\
\text { distal }\end{array}$ & $\begin{array}{c}\text { Região } \\
\text { proximal }\end{array}$ & $\begin{array}{c}\text { Região } \\
\text { distal }\end{array}$ & $\mathbf{p}$ \\
& (diencéfalo) & (telencéfalo) & (diencéfalo) & (telencéfalo) \\
\hline Indice de pulsatilidade & 1,55 & 1,69 & 1,73 & 1,86 & 0,38 \\
Índice de resistência & 0,77 & 0,79 & 0,83 & 0,79 & 0,29 \\
Relação sístole/diástole & 4,29 & 4,86 & 5,83 & 5,46 & 0,39 \\
\hline
\end{tabular}

Teste Mann-Whitney.

Os valores são medianas.

No grupo estudo compararam-se os exames normais $v s$ anormais (sem centralização $v s$ centralizados). Nos 15 fetos centralizados e/ou com CIUR, a mediana dos índices no diencéfalo (primeira medida) foi de $I P=1,02, I R=0,63$ e $S D=2,68 . \mathrm{Na}$ segunda medida (telencéfalo) a mediana foi de 
$\mathrm{IP}=0,95$, IR=062 e $\mathrm{SD}=2,44$. Nos fetos não centralizados, a mediana na primeira medida (diencéfalo) foi $\mathrm{IP}=1,15$, IR=0,66 e SD=3,25 (Tabela 2). Na segunda medida (telencéfalo) foi $\mathrm{IP}=1,31$, IR=0,70 e $\mathrm{SD}=3,70$ (Tabela 2). Nos fetos centralizados as diferenças no IP, IR e SD em função do local de análise (telencéfalo $v s$ diencéfalo) mostraram os seguintes valores de $\mathrm{p}$ : $\mathrm{IP}=0,53, \mathrm{IR}=0,56$ e $\mathrm{SD}=0,31$, portanto, sem significância estatística. Nos fetos sem centralização, as diferenças entre as medidas nos dois locais não mostraram ser significativas com os seguintes valores de $\mathrm{p}$ : $\mathrm{IP}=0,54, \mathrm{IR}=0,51$ e $\mathrm{SD}=0,36$.

Tabela 2 - Comparação dos locais dos índices dopplervelocimétricos na artéria cerebral no grupo estudo (DHEG; CIUR): exames normais vs anormais.

\begin{tabular}{|c|c|c|c|c|c|}
\hline \multirow{4}{*}{$\begin{array}{c}\text { Índices } \\
\text { dopplervelocimétricos }\end{array}$} & \multicolumn{5}{|c|}{ Grupo estudo } \\
\hline & \multicolumn{2}{|c|}{$\begin{array}{c}\text { Exames normais } \\
\qquad \mathrm{n}=35\end{array}$} & \multicolumn{3}{|c|}{$\begin{array}{c}\text { Anormais } \\
\text { (centralizados) } n=15\end{array}$} \\
\hline & $\begin{array}{c}\text { Região } \\
\text { proximal }\end{array}$ & $\begin{array}{l}\text { Região } \\
\text { distal }\end{array}$ & $\begin{array}{l}\text { Região } \\
\text { proximal }\end{array}$ & $\begin{array}{l}\text { Região } \\
\text { distal }\end{array}$ & $p$ \\
\hline & \multicolumn{5}{|c|}{ (diencéfalo)(telencéfalo)(diencéfalo)(telencéfalo) } \\
\hline Índice de pulsatilidade & 1,15 & 1,31 & 1,02 & 0,95 & 0,54 \\
\hline Índice de resistência & 0,66 & 0,70 & 0,63 & 0,62 & 0,51 \\
\hline Relação sístole/diástole & 3,25 & 3,70 & 2,68 & 2,44 & 0,36 \\
\hline
\end{tabular}

Teste Mann-Whitney.

Os valores são medianas.

\section{Discussão}

As comparações em dois grupos (pacientes normais $v s$ anormais) bem como as análises dentro de um mesmo grupo, em que fetos centralizados foram comparados com os não centralizados, permitiram avaliar a questão: se existem modificações da auto-regulação no fluxo cerebral em uma mesma população (fetos centralizados e não centralizados em portadoras de DHEG e/ou CIUR, grupo estudo).

No grupo estudo, verificou-se que a centralização e/ou CIUR estiveram presentes em 30\% dos fetos $(n=15)$. A literatura relata ser o CIUR fator que complica 5 a $10 \%$ de todas as gestações e acima de $30 \%$ das gemelares ${ }^{6}$. Em $60 \%$ destas, a causa principal do CIUR é a insuficiência placentária e grande parcela dos casos estão associados à hipertensão crônica e à DHEG. O presente trabalho mostrou que as placentas no grupo estudo apresentaram grau de amadurecimento precoce, como esperado em casos de hipertensão arterial crônica, DHEG ou CIUR.

A melhoria na identificação dos fetos com risco de morte intra-uterina pode conduzir a estratégias bem sucedidas de acompanhamento pré- natal. O presente estudo revelou que em uma mesma população (grupo estudo), ao se compararem fetos com centralização com os fetos não centralizados, não se observaram modificações estatisticamente significativas ao analisar os índices dopplervelocimétricos em função do local de insonação da artéria cerebral. A mediana dos índices IP, IR e SD neste subgrupo, na primeira medida (proximal), foi de respectivamente $\mathrm{IP}=1,02$, $\mathrm{IR}=0,63$ e $\mathrm{SD}=2,68$ e na segunda medida (distal) $\mathrm{IP}=1,31$, IR=0,70 e $\mathrm{SD}=3,70$, com os cálculos estatísticos não apontando significância (valor de $\mathrm{p}$ de 0,54 para o IP, 0,51 para o IR e de 0,36 para o SD).

Os índices dopplervelocimétricos são obtidos por meio da escolha do operador tanto do vaso a ser insonado como do local de obtenção da amostra. Na artéria cerebral média a auto-regulação do fluxo cerebral se faz em função da necessidade de maior ou menor aporte de oxigênio para os tecidos. A amplitude de pulso e velocidade de fluxo é quase linear em uma escala de valores com relação à perfusão cerebral e é afetada por mudanças na circulação do sistema nervoso central, bem como pela elasticidade dos tecidos intracranianos ${ }^{7}$. A artéria cerebral média é vaso relativamente longo e passa por regiões diferentes do cérebro, com diferentes resistências teciduais. Partindo destes pressupostos, realizou-se a avaliação da cerebral média em diferentes locais do cérebro (diencéfalo e telencéfalo). A Figura 1 mostra os locais de obtenção da amostra de fluxo na artéria cerebral média.
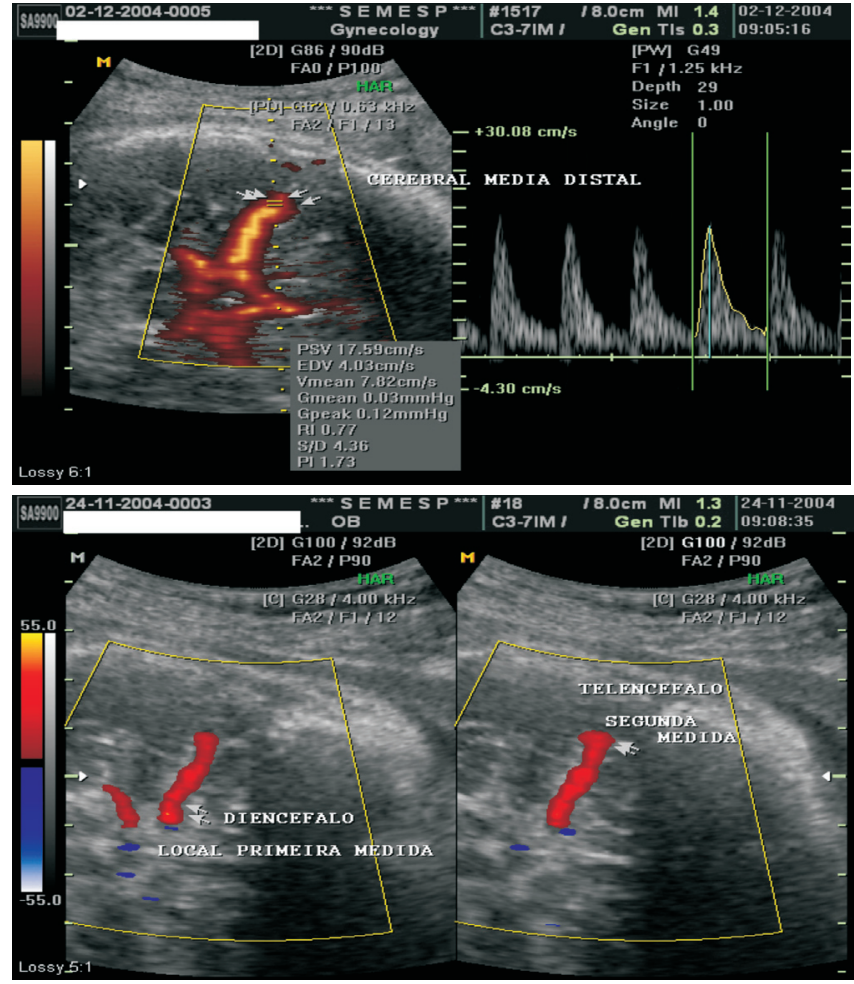

Figura 1 - Local de insonação da artéria cerebral média: diencéfalo x telencéfalo. 
Em casos de auto-regulação acentuada e não habitual, como exemplo nos fetos com centralização ou submetidos a ambiente intra-uterino não adequado onde exista insuficiência placentária (DHEG, hipertensas, CIUR), verificar se o local de obtenção dos índices interfere ou não nos resultados de suas medidas é oportuno para evitar falsopositivos e/ou negativos. A padronização do local de amostragem seria fundamental se existissem modificações nos valores em função de onde se obtêm os indices.

A artéria cerebral média é vaso exaustivamente estudado em correlações clínico-obstétricas. Zimmerman et al. ${ }^{8}$ demonstraram a importância do estudo da velocidade de pico sistólico na cerebral média com valor preditivo do grau de anemia fetal, em gestações antes da $34^{\mathrm{a}}$ semana, permitindo adequar e diminuir os procedimentos invasivos, no caso, a amniocentese e cordocentese. Assim, se os procedimentos invasivos puderem ser substituídos com testes não invasivos, a morbidade e a mortalidade fetal poderão ser reduzidas. Como as informações a respeito dos estudos de dopplervelocimetria no feto podem conduzir a decisões clínicas impróprias, é imperativo que as medidas e as técnicas sejam realizadas por operadores experientes e que não haja modificações relevantes nos índices ao longo do vaso estudado.

A literatura apresenta poucos trabalhos relacionando alterações dos índices de dopplervelocimetria com o local de obtenção das amostras, sendo alguns francamente discordantes, por não respeitarem alguns dos critérios citados neste estudo. Abel et al. ${ }^{9}$ sugeriram que existiam diferenças significativas ao se modificar o local de insonação nas artérias cerebrais ao avaliar 151 fetos entre 16 e 42 semanas de gestação. Existe variação importante nos índices dopplervelocimétricos em função da idade gestacional. Estes autores mediram o pico sistólico na região proximal e distal da artéria, realizando 4 medidas em cada feto. Modificações estatisticamente significantes foram observadas entre a região distal e a região proximal e não houve modificações em duas medidas no mesmo segmento, neste caso, no proximal. Concluíram que a velocidade de pico sistólico é afetada pela localização de insonação do vaso. Não descreveram parâmetros técnicos de angulação da insonação, e as variações dos índices de velocidade são grandes em função da idade gestacional. No presente estudo, o intervalo entre as idades gestacionais, sendo de apenas 6 semanas, diminui possiveis vieses.

Meyberg et al. ${ }^{10}$ avaliaram a curva de normalidade da artéria cerebral média em gravidez do terceiro trimestre e observaram que há progressivo aumento do componente diastólico por diminuição simultânea na resistência cerebral. A relação S/D na semana $29^{a}$ foi em média 8,0 , na $34^{\mathrm{a}}$ semana foi de 6,0 e na $40^{\mathrm{a}}$ semana de 3,5 . Houve um decréscimo no IR de 0,88 para 0,67. Outros autores demonstraram escalas similares, entretanto, os dados absolutos são diferentes. O aumento no componente diastólico na artéria cerebral média é observação importante, o que pode funcionar como fator de confusão em séries com grande variação da idade gestacional, como no trabalho citado anteriormente. O conhecimento da escala de referência pode ajudar a discriminar entre uma situação normal e a doença fetal. Por causa das escalas absolutas diferentes na literatura, cada centro perinatal deveria desenvolver seus próprios dados.

Hellmeyer et al. ${ }^{4}$ demonstraram haver discordâncias ao analisar as quatro artérias mais usadas em estudo obstétrico. Encontraram divergência de mais de $20 \%$ entre dois investigadores com as seguintes distribuições: artéria umbilical $16 \%$, artéria cerebral média $42 \%$, artéria uterina direita $28 \%$ e artéria uterina esquerda $37 \%$. Concluíram que a variabilidade interobservador era surpreendentemente elevada e a correlação aceitável poderia ser indicada somente para a artéria umbilical, e que o uso rotineiro de equipamentos e operadores diferentes deve ser evitado. Cuidadosa interpretação do exame associada à clínica é imperativa. O presente estudo eliminou o efeito interobservador porque todos os exames foram realizados pelo mesmo profissional ${ }^{11}$.

Os valores descritos na literatura para a fase da gestação estão de acordo com os encontrados no nosso estudo. Aranyosi et al. ${ }^{12}$ demonstraram em gravidezes normais de 28 a 41 semanas, um valor para o IP de $1,7 \pm 0,4$ e ao nascimento de $1,3 \pm 0,5$ e para o IR média de $0,64 \pm 0,12$.

No presente estudo obtivemos boa padronização dos grupos e amostra adequada onde foi estudado número significativo de fetos. Ressalta-se intervalo gestacional menor, a presença de um grupo controle ao de doenças, permitindo assim, confiabilidade nos resultados apresentados. Os indices dopplervelocimétricos, quando se trata de vários operadores, estão sujeitos a maiores erros, visto que estão em função do ângulo de insonação bem como da medida correta do diâmetro do vaso e da qualidade do aparelho, portanto, muito provavelmente associados a viés.

A correção do ângulo realizada na técnica descrita em material e métodos para a obtenção dos indices está de acordo com a literatura, que o considera ideal, o mais longe possivel de $90^{\circ}$, por- 
tanto, na nossa série, realizamos esta correção de $0^{\circ}$ a $10^{\circ}$ bem como usamos um intervalo menor, como forma de dar segurança aos índices obtidos e facilitar a padronização das amostras.

Há de se considerar em aplicação prática que se a artéria umbilical apresentar diástole cheia e indices normais, associadas com outros padrões de boa vitalidade fetal, como o liquido amniótico e a presença de tônus normal e movimentos respiratórios, as variações na artéria cerebral média para baixo da normalidade não estão associadas a repercussões clínicas fetais.

Concluímos pelos resultados apresentados que o uso de diferentes pontos para o estudo dopplervelocimétrico na artéria cerebral média não interfere na obtenção dos índices de resistência, de pulsatilidade e na relação sístole/diástole. O estudo sugere que a localização não deve constituir preocupação do operador.

\section{Referências}

1. Demianczuk NN, Van Den Hof MC, Farquharson D, Lewthwaite B, Gagnon R, Morin L, et al. The use of first trimester ultrasound. J Obstet Gynaecol Can. 2003;25(10):864-75.

2. Skotnicki MZ, Flig E, Urban J, Hermann T. Doppler examinations in the prognosis of birth status of the newborn. Med Sci Monit. 2000;6(3):611-5.

3. Berger R, Garnier Y. Perinatal brain injury. J Perinat Med. 2000;28(4):261-85.

4. Hellmeyer L, Sierra F, Heller G, Tekesin I, Kuhnert M, Schmidt S. Evaluation of the reproducibility of Doppler ultrasonographic measurements in obstetrics. Z Geburtshilfe Neonatol. 2003;207(4):127-31.
5. Centers for Disease Control and Prevention (CDC). Epi Info [computer program]. Version 3.3. Atlanta (GA): CDC; 2004.

6. Aucott SW, Donohue PK, Northington FJ. Increased morbidity in severe early intrauterine growth restriction. J Perinatol. 2004;24(7):435-40.

7. Ursino M, Giulioni M. Quantitative assessment of cerebral autoregulation from transcranial Doppler pulsatility: a computer simulation study. Med Eng Phys. 2003;25(8):655-66.

8. Zimmerman R, Carpenter RJ Jr, Durig P, Mari G. Longitudinal measurement of peak systolic velocity in the fetal middle cerebral artery for monitoring pregnancies complicated by red cell alloimmunisation: a prospective multicentre trial with intention-to-treat. BJOG. 2002;109(7):746-52.

9. Abel DE, Grambow SC, Brancazio LR, Hertzberg BS. Ultrasound assessment of the fetal middle cerebral artery peak systolic velocity: a comparison of the near-field versus far-field vessel. Am J Obstet Gynecol. 2003;189(4):986-9.

10. Meyberg R, Ertan AK, Tossounidis I, Friedrich M, Schmidt W. Reference ranges and standard percentile-curves for the Doppler indices RI and S/D ratio of the fetal middle cerebral artery. Color Doppler measurements in a perinatal centre. Clin Exp Obstet Gynecol. 2000;27(2):106-8.

11.Schoning M, Scheel P. Color duplex measurement of cerebral blood flow volume: intra- and interobserver reproducibility and habituation to serial measurements in normal subjects. J Cereb Blood Flow Metab. 1996;16(3):523-31.

12.Aranyosi J, Bettembuk P, Zatik J, Ovari L, Torok I, Godeny S. Doppler evaluation of the fetal arterial circulation: reference values of the Resistance Index and Pulsatility Index between the 28th and 41st weeks of gestation. Orv Hetil. 2001;142(34):184750. 